\title{
Micromechanics Modeling of Composites Subjected to Multiaxial Progressive Damage in the Constituents
}

\author{
Brett A. Bednarcyk* \\ NASA John H. Glenn Research Center at Lewis Field, Cleveland, Ohio 44135

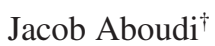 \\ Tel-Aviv University, 69978 Ramat-Aviv, Israel \\ and \\ Steven M. Arnold \\ NASA John H. Glenn Research Center at Lewis Field, Cleveland, Ohio 44135
}

DOI: $\underline{10.2514 / 1.45671}$

\begin{abstract}
The high-fidelity generalized method of cells composite micromechanics model is extended to include constituentscale progressive damage via a proposed damage model. The damage model assumes that all material nonlinearity is due to damage in the form of reduced stiffness, and it uses six scalar damage variables (three for tension and three for compression) to track the damage. Damage strains are introduced that account for interaction among the strain components and that also allow the development of the damage evolution equations based on the constituent material uniaxial stress-strain response. Local final-failure criteria are also proposed based on mode-specific strain energy release rates and total dissipated strain energy. The coupled micromechanics-damage model described herein is applied to a unidirectional $\boldsymbol{E}$-glass/epoxy composite and a proprietary polymer matrix composite. Results illustrate the capability of the coupled model to capture the vastly different character of the monolithic (neat) resin matrix and the composite in response to far-field tension, compression, and shear loading.
\end{abstract}

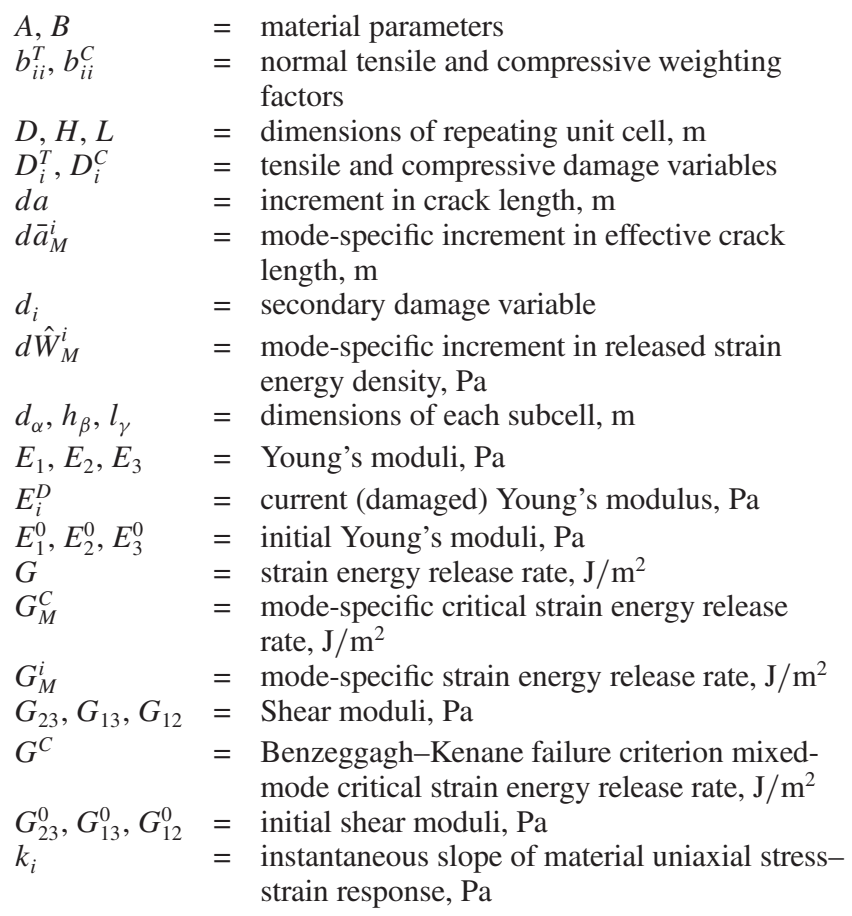

Received 26 May 2009; revision received 28 March 2010; accepted for publication 13 April 2010. This material is declared a work of the U.S. Government and is not subject to copyright protection in the United States. Copies of this paper may be made for personal or internal use, on condition that the copier pay the $\$ 10.00$ per-copy fee to the Copyright Clearance Center, Inc., 222 Rosewood Drive, Danvers, MA 01923; include the code 0001-1452/ 10 and $\$ 10.00$ in correspondence with the CCC.

*Materials Research Engineer, 21000 Brookpark Road; Brett.A. Bednarcyk@nasa.gov. AIAA Senior Member.

${ }^{\dagger}$ Professor, School of Mechanical Engineering.

ॠChief, Mechanics and Life Prediction Branch, 21000 Brookpark Road; AIAA Member.

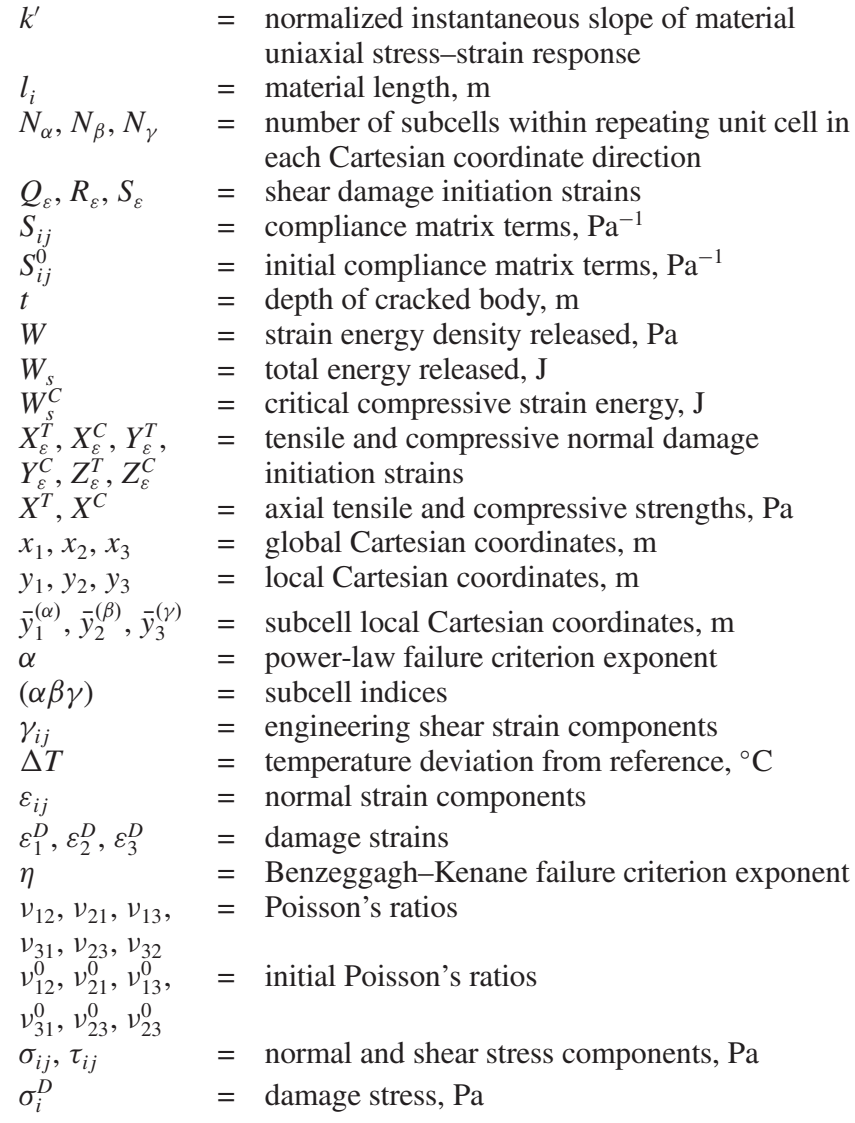

\section{Introduction}

$\mathbf{T}$ HE importance of capturing the progressive nature of failure in polymer matrix composites was highlighted by the World-Wide Failure Exercise (WWFE) [1]. While many ply-level failure criteria performed reasonably well when damage initiation was followed closely by final failure, in situations where significant nonlinearity 
occurred before final failure, most standard criteria were insufficient. Methods that capture this nonlinearity have shown much better agreement with experimental data from the WWFE (cf., Bogetti et al. [2] and Nelson et al. []ㅡ).

In the present paper, the high-fidelity generalized method of cells (HFGMC) micromechanics model $[\underline{4}, \underline{5}]$ is extended via an elastic progressive damage model to predict progressive failure of polymer matrix composites. This work was motivated by previous efforts [6] that indicated that HFGMC's damage modeling capabilities were insufficient. The damage model employed previously within HFGMC relied on typical failure criteria (e.g., maximum stress, maximum strain, Tsai-Hill, and Tsai-Wu) to predict local failure within the subvolumes (subcells) within the HFGMC composite, repeating unit cell (RUC) based on the local (constituent level) stress-strain state. Then, upon failure, all of the subvolume's stiffness components were instantaneously reduced to a very low value. The damage progression was thus modeled as a series of these instantaneous subvolume failures before final failure of the composite or laminate. This previous work [6] revealed two major shortcomings of the approach:

1) The damage evolution (essentially a step function) was not sufficient to capture the progressive nature of the failure, particularly in shear dominated cases.

2) The single damage parameter that affected all stiffness components equally (i.e., lack of multiaxiality) was insufficient to capture the vastly different behavior observed in tension, compression, and shear.

To overcome these deficiencies, a damage model is proposed herein that accounts for the multiaxiality and progressive nature of damage within the constituent materials as simply as possible. It is thus assumed that all nonlinearity exhibited by the composite is due to damage, resulting in stiffness reduction, within the constituent materials. To account for differences in the response in tension, compression, and shear, three scalar damage variables and three damage strains are tracked in tension and three in compression. The effects of the damage variables on the material shear stiffness components can be controlled by additional material parameters. The damage strains are related to the orientation of the damage on the constituent scale and, assuming that these damage strains correctly capture the interaction effects among the strain components, it is possible to determine the evolution equations for the damage variables based on uniaxial stress-strain curves for the constituent materials.

One way for a subvolume (subcell) within the composite to reach final failure in a given direction is for the damage to evolve until the subvolume stiffness in a given direction is close to zero. However, it is desirable to enable subcells to fail suddenly after a certain amount of damage has accumulated. As such, final tensile and shear failure criteria are introduced based on the mode-specific strain energy release rates, which can be calculated due to the directionality of the damage in the proposed model. For compression, a total dissipated strain energy criterion is considered.

The proposed damage model uses the continuum damage approach first introduced by Kachanov [7,8], in which continuous damage variables are introduced to quantify the stiffness reduction of a material. The theoretical background of this approach, and specifically, the elastic damage approach, is discussed in detail by Lemaitre and Chaboche [9], Krajcinovic [10], Voyiadjis and Kattan [11], and Talreja [12,13]. One particular anisotropic elastic continuum damage model that has received a good deal of attention in recent years and is similar in some ways to that proposed herein is that of Matzenmiller et al. [14]. This model has been incorporated within LS-DYNA $[\underline{15}, \underline{16}]$ as a progressive damage constitutive model for shell elements. The shell element progressive damage model available in ABAQUS [17] also uses an approach similar to Matzenmiller et al. [14], although the shear damage variable and damage evolution equations are different.

Like the model of Matzenmiller et al. [14], the proposed damage model considers anisotropic elastic damage using a number of scalar damage parameters. Both models also track tensile and compressive damage separately. The model of Matzenmiller et al. [14] was intended to simulate the response of an anisotropic composite ply, and thus considers plane stress conditions. The model proposed herein is intended for application to the constituents within a composite material, and thus considers a three-dimensional state of stress and strain. The damage initiation criteria employed by Matzenmiller et al. [14] are based on the Hashin [18] stress-based failure criteria, while the proposed model uses three-dimensional strain-based Hashin-like damage initiation criteria. Matzenmiller et al. [14] also include an independent in-plane shear damage parameter, while the model described herein relates the shear damage parameters to the normal damage parameters. Finally, the damage evolution equations and final-failure criteria of the two models are distinct. It is also noted that, like the model of Matzenmiller et al. [14], the proposed damage model could be readily implemented within finite-element models, including ABAQUS [17] and LSDYNA [15], through their user material subroutines (although not done at this time). The present modular implementation of the model has been incorporated within NASA's Micromechanics Analysis Code with Generalized Method of Cells (MAC/GMC) [19].

After the micromechanics theory and the proposed damage model are described, the coupled micromechanics-damage approach is applied to simulate a unidirectional $E$-glass/epoxy composite, for which the constituents are characterized based on ply-level data from the literature [1]. The model's ability to capture vastly different damage behavior in tension, compression, and shear is highlighted as monolithic and composite nonlinear stress-strain curves, and damage initiation and final-failure envelopes are presented.

\section{Micromechanics Model}

The HFGMC micromechanics model has been adopted to model the composite-level behavior in this paper. The HFGMC model is based on a homogenization technique for composites with periodic microstructure, as shown in Fig. 1a, in terms of the global coordinates $\left(x_{1}, x_{2}, x_{3}\right)$. The parallelepiped RUC (Fig. 1b) defined with respect to the local coordinates $\left(y_{1}, y_{2}, y_{3}\right)$ of such a composite is divided into $N_{\alpha}, N_{\beta}$, and $N_{\gamma}$ subcells in the $y_{1}, y_{2}$, and $y_{3}$ directions, respectively. Each subcell is labeled by the indices $(\alpha \beta \gamma)$ with $\alpha=1, \ldots, N_{\alpha}$, $\beta=1, \ldots, N_{\beta}$, and $\gamma=1, \ldots, N_{\gamma}$ and may contain a distinct homogeneous material. The dimensions of the subcell are denoted by $d_{\alpha}, h_{\beta}$, and $l_{\gamma}$, respectively. A brief outline of the HFGMC method is provided next. The reader is referred to Aboudi [4] for the full theoretical development in the case of linear electromagnetothermoelastic materials and Aboudi et al. [20,21] for the twodimensional case of continuous fibers including inelasticity of the phases.

1) A quadratic displacement field is assumed for each subcell in terms of the local subcell coordinates $\left(\bar{y}_{1}^{(\alpha)}, \bar{y}_{2}^{(\beta)}\right.$, and $\bar{y}_{3}^{(\gamma)}$ in Fig. 1) and 21 unknown coefficients.

2) Equilibrium conditions, periodic boundary conditions, and interfacial continuity conditions of displacements and tractions between subcells are imposed in the average (integral) sense.

3 ) The result is a system of linear algebraic equations, which is solved for the 21 unknown coefficients per subcell in terms of the constituent material properties, microstructural dimensions, and thermal and inelastic terms.

4) Knowledge of these coefficients enables establishment of a localization relation that relates the average strain in each subcell to the externally applied average strain [5] , along with thermal and inelastic terms.

4) The local thermoinelastic constitutive equations are used in conjunction with the average stress relations to arrive at the effective thermoinelastic constitutive equations for the heterogeneous material.

As stated, the original version of HFGMC requires solution of a system of $21 N_{\alpha} N_{\beta} N_{\gamma}$ algebraic equations. However, just like the reformulation of generalized method of cells micromechanics model that has been presented by Pindera and Bednarcyk [22], it is possible in the case of perfect bonding between the phases to now use the continuity of displacements across the interfaces between the phases and significantly reduce the number of equations that have to be 


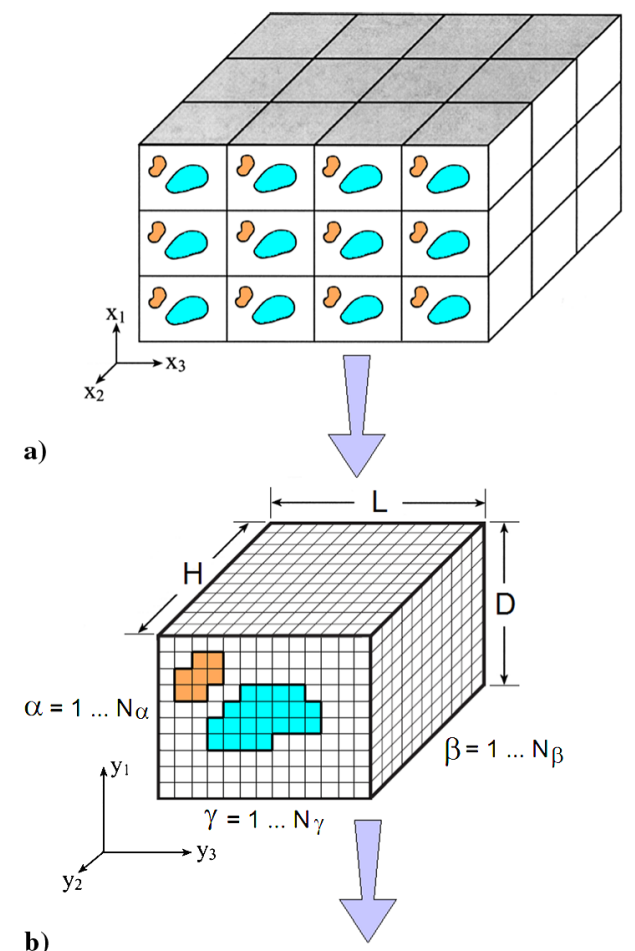

b)

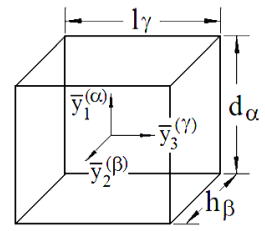

c)

Fig. 1 HFGMC model: a) a multiphase composite with triply periodic microstructures, b) the RUC, defined in the $\left(y_{1}, y_{2}, y_{3}\right)$ coordinate system, is discretized into $N_{\alpha} \times N_{\beta} \times N_{\gamma}$ subcells, and c) the monolithic subcell is defined in the local coordinate system $\left(\bar{y}_{1}^{(\alpha)}, \bar{y}_{2}^{(\beta)}, \bar{y}_{3}^{(\gamma)}\right)$.

solved. This reformulation of HFGMC has been presented by Bansal and Pindera [23] in the special case of continuous fibers (i.e., the twodimensional, doubly periodic case) and elastic phases, with the more general case presented by Arnold et al. [24] The unknowns in this reformulation are given by the average displacements at the surfaces of the subcells, which can be related to the original HFGMC unknown coefficients. However, by formulating the HFGMC theory in terms of the subcell surface average displacements, the size of the system of algebraic equations that must be solved becomes

$$
9 N_{\alpha} N_{\beta} N_{\gamma}+3\left(N_{\alpha} N_{\beta}+N_{\alpha} N_{\gamma}+N_{\beta} N_{\gamma}\right)
$$

rather than $21 N_{\alpha} N_{\beta} N_{\gamma}$, with an obvious significant savings. The ramifications of these savings are discussed in detail by Arnold et al. [24].

More recently, Haj-Ali and Aboudi [25] formulated the HFGMC model in a new form that facilitates its computational efficiency. To this end, the explicit matrix form of the HFGMC model was presented, which allowed an immediate and convenient computer implementation of the model. It was shown that a dramatic reduction of the computational effort can be achieved by performing a condensation procedure that, for the doubly periodic HFGMC, reduces the dimension of the condensed system of equation to $6 N_{\beta} N_{\gamma} \times 6 N_{\beta} N_{\gamma}$, just like the reformulation discussed previously.

\section{Progressive Damage Model}

A progressive damage model is sought to function within the HFGMC micromechanics model at the level of the fiber/matrix constituents. The two significant advances beyond the damage models previously employed within HFGMC (cf., Moncada et al. [6]) are 1) multiaxiality, such that the response in normal tension, normal compression, and shear may be independent, and 2) progression, such that the damage can occur gradually rather than instantaneously. It is also desirable to accomplish these goals as simply as possible and while requiring as few model parameters as possible. As such, an irreversible elastic damage model is proposed that attributes all material nonlinearity to a reduction in material stiffness properties.

The elastic continuum damage model tracks the damage in a material element through six scalar damage variables: $D_{i}^{T}$ and $D_{i}^{C}$, $i=1,2,3$. Each damage variable is associated with damage oriented normal to the Cartesian coordinate direction indicated by the subscript, and separate damage variables track tensile $T$ and compressive $C$ damage. While these damage variables may be written in vector form, the model is not a vector damage model but rather a multiscalar approach, as the damage parameters do not necessarily adhere to first-order tensorial mathematics.

It is assumed that the damage variables modify the engineering material properties rather than the stresses or strains, and those in the undamaged state, $D_{i}^{T}=D_{i}^{C}=0$, while the completely damaged (final failure) state corresponds to $D_{i}^{T}=1$ or $D_{i}^{C}=1$. The dependence of the engineering material properties on the damage variables is taken as

$$
\begin{aligned}
E_{1}=d_{1} E_{1}^{0} ; \quad E_{2}=d_{2} E_{2}^{0} ; \quad E_{3}=d_{3} E_{3}^{0} ; & v_{12}=d_{1} v_{12}^{0} ; \\
v_{13}=d_{1} v_{13}^{0} ; & v_{31}^{0}=d_{3} v_{31}^{0} ; \\
v_{23}=d_{2} v_{23}^{0} ; & v_{32}=d_{3} v_{23}^{0}
\end{aligned}
$$

where,

$$
d_{i}= \begin{cases}1-b_{i i}^{T} D_{i}^{T} & \sigma_{i i}>0 \\ 1-b_{i i}^{C} D_{i}^{C} & \sigma_{i i}<0\end{cases}
$$

$\sigma_{i i}, i=1,2,3$ are the normal stress components (no summation), $E_{1}^{0}$, $E_{2}^{0}$, and $E_{3}^{0}$ are the initial material Young's moduli, $v_{12}^{0}, v_{21}^{0}, v_{13}^{0}, v_{31}^{0}$, $v_{23}^{0}$, and $v_{32}^{0}$ are the initial material Poisson ratios, and the corresponding quantities without the 0 superscript are the current (damaged) material properties. The $b_{i i}^{T}$ and $b_{i i}^{C}$ terms (no summation) are constants that control the dependence of the damaged moduli on the appropriate damage variables and thus enable scaling of this dependence between tension and compression. The default value of these $b$ terms is one, and the minimum allowable value of $d_{i}$ is zero, but in practice, a small positive minimum value of $d_{i}$ (such as 0.0001 ) is employed to promote stability of model simulations. Note that, because the Young's moduli are affected independently by the damage variables, a constituent material that is initially isotropic becomes orthotropic upon damage initiation.

It is assumed that the shear moduli dependence on the damage variables is given by a linear combination of the appropriate two damage variables:

$$
\begin{aligned}
G_{23} & =\left(1-b_{42} D_{2}^{\bullet}-b_{43} D_{3}^{\bullet}\right) G_{23}^{0} ; \\
G_{13} & =\left(1-b_{51} D_{1}^{\bullet}-b_{53} D_{3}^{\bullet}\right) G_{13}^{0} ; \\
G_{12} & =\left(1-b_{61} D_{1}^{\bullet}-b_{62} D_{2}^{\bullet}\right) G_{12}^{0}
\end{aligned}
$$

where

$$
D_{i}^{\bullet}= \begin{cases}D_{i}^{T} & \sigma_{i i}>0 \\ D_{i}^{C} & \sigma_{i i}<0\end{cases}
$$

and $G_{23}^{0}, G_{13}^{0}$, and $G_{12}^{0}$ are the initial material shear moduli. The $b$ terms again enable scaling of the damage variable dependence of the material properties, and the shear related $b$ terms in Eq. (3) have default values of 0.5 . The factors that modify the initial shear moduli in Eq. (3) (e.g., $1-b_{42} D_{2}^{\bullet}-b_{43} D_{3}^{\bullet}$ ), have a minimum allowable value of zero, but in practice, a small positive minimum value (such as 0.0001 ) is employed to promote stability. 
It is noted that the dependence of the damaged Poisson ratios on the damage variables [Eq. (1)] preserves the symmetry of the material compliance matrix. For example, denoting the compliance matrix components by $S_{i j}$ :

$$
S_{12}=-\frac{\nu_{21}}{E_{2}}=S_{21}=-\frac{\nu_{12}}{E_{1}}
$$

Substituting for the material properties using Eq. (1),

$$
-\frac{d_{2} \nu_{21}^{0}}{d_{2} E_{2}^{0}}=-\frac{d_{1} \nu_{12}^{0}}{d_{1} E_{1}^{0}}=S_{12}^{0}=S_{21}^{0}
$$

and, as shown, since the initial compliance matrix is symmetric, it is clear that the damaged compliance matrix will remain symmetric as well. In addition, $S_{i j}=S_{i j}^{0}, i \neq j$, so the damage only affects the diagonal terms of the compliance matrix. Finally, this form of damage for the Poisson ratios is mechanistically correct. That is, if one considers a typical Poisson mechanical test where, for example, $\sigma_{11} \neq 0$, and all other stress components are equal to zero, we have by definition

$$
v_{12}=-\frac{\varepsilon_{22}}{\varepsilon_{11}}
$$

where $\varepsilon_{i j}$ are the strain components. Thus, considering the case of damage only in the $x_{1}$ direction $\left(d_{1} \rightarrow 0\right)$, one would expect a small $\varepsilon_{22}$ and a large $\varepsilon_{11}$, leading to a small $v_{12}$. According to Eqs. (1) and (2), it is clear that the preceding case corresponds to a low value of $d_{1}$ and, thus, a small $v_{12}$. Now, considering a constrained mechanical test where, for example, $\sigma_{11} \neq 0$ is applied, $\varepsilon_{22}=0$ is constrained, and $\sigma_{22} \neq 0$ with all other stress components equal to zero, we have

$$
S_{21} \sigma_{11}+S_{22} \sigma_{22}=\varepsilon_{22}=0
$$

Thus, substituting using Eqs. (1) and (ㅁ),

$$
\sigma_{22}=-\frac{S_{21}}{S_{22}} \sigma_{11}=\frac{E_{2} v_{12}}{E_{1}} \sigma_{11}=\frac{d_{2} E_{2}^{0} d_{1} v_{12}^{0}}{d_{1} E_{1}^{0}} \sigma_{11}=d_{2} \frac{E_{2}^{0} \nu_{12}^{0}}{E_{1}^{0}} \sigma_{11}
$$

Therefore, in the case of damage in the $x_{2}$ direction $\left(d_{2} \rightarrow 0\right)$, we see that the material loses the ability to accumulate normal stress in the $x_{2}$ direction, regardless of the damage in the $x_{1}$ direction.

\section{A. Damage Initiation}

The damage initiation criterion is taken as a three-dimensional extended version of the strain-based Hashin [18] criterion. The following damage strains are defined,

$$
\begin{aligned}
& \varepsilon_{1}^{D}=\sqrt{\left(\frac{\varepsilon_{11}}{X_{\varepsilon}}\right)^{2}+\left(\frac{\gamma_{13}}{R_{\varepsilon}}\right)^{2}+\left(\frac{\gamma_{12}}{S_{\varepsilon}}\right)^{2}} ; \\
& \varepsilon_{2}^{D}=\sqrt{\left(\frac{\varepsilon_{22}}{Y_{\varepsilon}}\right)^{2}+\left(\frac{\gamma_{23}}{Q_{\varepsilon}}\right)^{2}+\left(\frac{\gamma_{12}}{S_{\varepsilon}}\right)^{2}} ; \\
& \varepsilon_{3}^{D}=\sqrt{\left(\frac{\varepsilon_{33}}{Z_{\varepsilon}}\right)^{2}+\left(\frac{\gamma_{23}}{Q_{\varepsilon}}\right)^{2}+\left(\frac{\gamma_{13}}{R_{\varepsilon}}\right)^{2}}
\end{aligned}
$$

where

$$
\begin{gathered}
X_{\varepsilon}=\left\{\begin{array}{cc}
X_{\varepsilon}^{T} & \sigma_{11}>0 \\
X_{\varepsilon}^{C} & \sigma_{11}<0
\end{array} ; \quad Y_{\varepsilon}=\left\{\begin{array}{ll}
Y_{\varepsilon}^{T} & \sigma_{22}>0 \\
Y_{\varepsilon}^{C} & \sigma_{22}<0
\end{array} ;\right.\right. \\
Z_{\varepsilon}= \begin{cases}Z_{\varepsilon}^{T} & \sigma_{33}>0 \\
Z_{\varepsilon}^{C} & \sigma_{33}<0\end{cases}
\end{gathered}
$$

$\varepsilon_{i i}, i=1,2,3$, are the normal stress components (no summation), $\gamma_{23}$, $\gamma_{13}$, and $\gamma_{12}$ are the engineering shear strains, $X_{\varepsilon}^{T}$ and $X_{\varepsilon}^{C}$ are the tensile and compressive damage initiation strains in the $x_{1}$ coordinate direction, $Y_{\varepsilon}^{T}$ and $Y_{\varepsilon}^{C}$ are the tensile and compressive damage initiation strains in the $x_{2}$-coordinate direction, $Z_{\varepsilon}^{T}$ and $Z_{\varepsilon}^{C}$ are the tensile and compressive damage initiation strains in the $x_{3}$-coordinate direction, and $Q_{\varepsilon}, R_{\varepsilon}$, and $S_{\varepsilon}$ are the damage initiation engineering shear strains associated with $\gamma_{23}, \gamma_{13}$, and $\gamma_{12}$, respectively. Thus, according to Eq. (10), damage will initiate when $\varepsilon_{1}^{D}, \varepsilon_{2}^{D}$, or $\varepsilon_{3}^{D}$ exceed one.

\section{B. Damage Evolution}

The fundamental tenet of the proposed irreversible elastic damage model is that the damage evolution is controlled by the damage strains, as defined in Eq. (10). That is, while the damage evolution controlled by these damage strains can be fit to reproduce uniaxial material behavior, it is an assumption of the proposed theory that the generalization to multiaxiality can be represented by the three damage strains defined in Eq. (10). This is, in many ways, analogous to the role of the second stress invariant in von Mises plasticity theory, which defines the multiaxial initiation (yield) condition and controls the evolution (flow).

Assuming that the loading imposed on the damaging material is done incrementally, as indicated by the dots in Fig. 2, it is possible to determine the damage variable increments from the damage strain components and damage strain increments given the instantaneous slope, $k_{i}$, of the material's uniaxial stress-strain response curve. Thus, the uniaxial material stress-strain response curve can be used to characterize the damage evolution through the damage initiation strain and the instantaneous slope. In the uniaxial normal loading case with loading in the $x_{i}$ direction, the damage strain reduces to $\varepsilon_{i}^{D}=\varepsilon_{i i} / X_{\varepsilon}$, and a corresponding damage stress, $\sigma_{i}^{D}$, which is linearly related to the damage strain by the damaged modulus, $E_{i}^{D}$, can be identified. Figure 2 graphically shows the relationship for the case normal uniaxial loading.

Because the loading is uniaxial, the modulus at any loading increment point is related to the initial modulus $E_{i}^{0}$ by the appropriate damage variable $D_{i}$ :

$$
E_{i}^{D}=\left(1-D_{i}\right) E_{i}^{0}
$$

The incremental change in the modulus can then be determined as

$$
d E_{i}^{D}=-d D_{i} E_{i}^{0}
$$

and the damage variable increment is then given by

$$
d D_{i}=-\frac{d E_{i}^{D}}{E_{i}^{0}}
$$

The damage stress is defined via a linear relationship with the damage strain that, for the uniaxial loading, is given by

$$
\sigma_{i}^{D}=E_{i}^{D} \varepsilon_{i}^{D}
$$

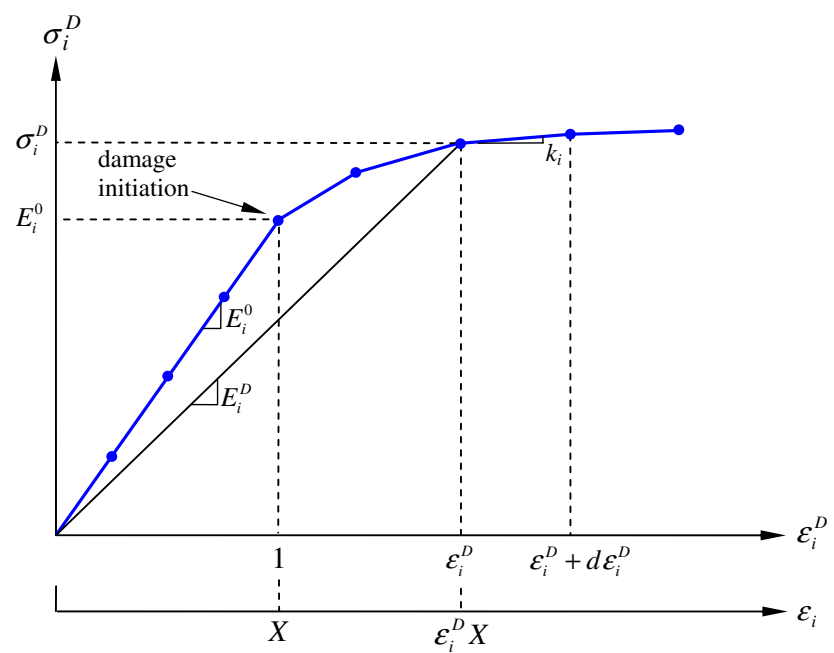

Fig. 2 An incremental elastic damage stress vs damage strain curve. Note that $X=X_{\varepsilon}, Y_{\varepsilon}$, or $Z_{\varepsilon}$. 
Note that, even in the uniaxial case, Poisson effects will give rise to damage strains in the remaining two normal directions according to Eq. (10). However, these will be much smaller than the damage strain in the applied loading direction and typically will not exceed one (which would indicate damage initiation in these directions) before final failure in the applied loading direction.

From Eq. (15), the incremental change in damage stress is given by

$$
d \sigma_{i}^{D}=E_{i}^{D} d \varepsilon_{i}^{D}+\varepsilon_{i}^{D} d E_{i}^{D}
$$

The instantaneous slope of the damage stress vs damage strain curve, which is equivalent to the instantaneous slope of the uniaxial stressstrain curve, $k_{i}$, is given by

$$
\frac{\mathrm{d} \sigma_{i}^{D}}{\mathrm{~d} \varepsilon_{i}^{D}}=E_{i}^{D}+\varepsilon_{i}^{D} \frac{\mathrm{d} E_{i}^{D}}{\mathrm{~d} \varepsilon_{i}^{D}}=k_{i}
$$

Isolating the incremental change in the damaged modulus gives

$$
d E_{i}^{D}=\left(k_{i}-E_{i}^{D}\right) \frac{d \varepsilon_{i}^{D}}{\varepsilon_{i}^{D}}
$$

and substituting Eq. (18) into Eq. (14) yields

$$
d D_{i}=\left(\frac{E_{i}^{D}-k_{i}}{E_{i}^{0}}\right) \frac{d \varepsilon_{i}^{D}}{\varepsilon_{i}^{D}}
$$

Substituting Eq. (12) into Eq. (19) and letting $k_{i}^{\prime}=k_{i} / E_{i}^{0}$ provides the equation for the damage variable evolution:

$$
d D_{i}=\left(1-D_{i}-k_{i}^{\prime}\right) \frac{d \varepsilon_{i}^{D}}{\varepsilon_{i}^{D}}
$$

Thus, given the fact that the damage initiates when $D_{i}=0$, along with the knowledge of the shape (i.e., instantaneous slope) of the postinitiation uniaxial stress-strain curve, the incremental damage evolution can be determined from Eq. (20). A uniaxial shear stressstrain curve could also be used and, if both normal and shear curves are available, they can be used to estimate the value of the $b$ terms in Eqs. (2) and (3). Otherwise, the default values may be assumed.

\section{Unloading, Reloading, and Load Reversals}

It is assumed that, upon unloading, the material immediately ceases to damage. That is, the damage strain state must be on a damage surface in order for further damage to occur. As such, the maximum previous value of each damage strain must be tracked and further damage only permitted when the previous maximum is exceeded. Because the model is elastic, unloading will always occur linearly to the zero stress-strain state. It is assumed that entirely different damage mechanisms are at work in tension and in compression. For instance, tensile damage might be due to microcracking, while in compression, it might be due to a microbuckling mechanism. This implies that both tensile and compressive damage strains must be tracked independently, with each causing the evolution of its corresponding tensile or compressive damage increment, according to Eq. (20). The previous maximum of the damage strains in both tension and compression must also be tracked. The result of these assumptions is that tensile damage will not affect compressive damage, and vice versa. Thus, if the material is subjected to a load reversal, the applicable damage variable will switch instantaneously, as will the apparent stiffness of the material (assuming damage has initiated). For example, if the material is first damaged in tension, unloaded, and then subjected to compression, the apparent stiffness will change instantaneously to the initial stiffness when the material goes into compression.

The behavior described previously is illustrated in Fig. 3a. The material is first loaded in normal tension. The behavior is linear until damage initiates at a strain of 0.0125 . The postdamage slope is negative, and the stress decreases in step 2 as the strain increases. In step 3, the material is unloaded, and the response is linear to the origin with a reduced (damaged) stiffness. Upon crossing the origin, the material is placed in compression in step 4 and, since the material is as yet undamaged in compression, the material returns to its original stiffness. Compressive damage initiates at a strain of -0.0287 , and the compressive postdamage slope in step 5 is positive. The material is unloaded in compression in step 6, and the response is again to the origin along a path with a reduced (damaged) stiffness. Upon reentering the tensile regime in step 7 , the material retraces its previous tensile unloading path, as the material has already been damaged in tension. Further damage does not occur until the previously attained maximum strain has been reached, at which point, in step 8, the material continues to damage. Clearly, vastly different nonlinear material tensile and compressive behavior can be accommodated by the proposed damage model.

In contrast, example material shear behavior is shown in Fig. $\underline{3 b}$. Steps 1-3 are similar, but it is clear that in shear (as there is no distinction between tension and compression) there is no change in slope when traversing from positive to negative stress. Further, additional damage in negative shear begins to accumulate when the previous maximum positive shear strain (magnitude) is reached. When reentering the tensile regime, there is again no change in slope, and the previous maximum negative shear strain (magnitude) is exceeded before additional damage can occur. Note that Figs. $3 \mathrm{a}$ and $3 \mathrm{~b}$ are based on the epoxy resin material properties for which the characterization is discussed in Sec. IV.

\section{Final Failure}

Final failure is considered to be the state at which the material can no longer support one or more components of stress in tension,
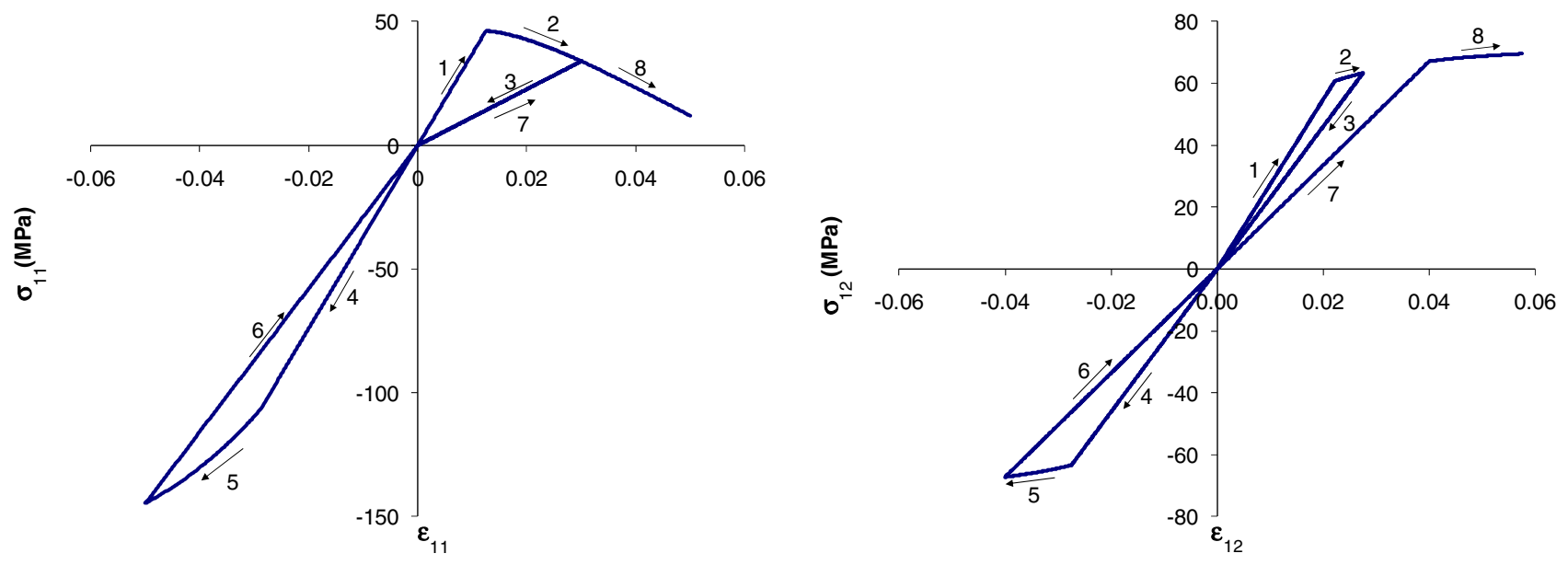

a)

b)

Fig. 3 Monolithic material described by the proposed damage model: a) cyclic tensile and compressive behavior and b) cyclic shear behavior. 
compression, or shear to any significant degree. At this point, the effective stiffness of the material, rather than being determined according to Eqs. (1) or (3), will be set to a very low value (i.e., 0.0001 times its initial value). One way that the material can reach final failure is by one of its damage variables evolving to the point that one of the stiffnesses reaches zero. It is desirable, however, to enable the material to reach final failure suddenly before a stiffness is evolving all the way to zero.

Separate final-failure criteria are proposed for tension and compression. In tension, a strain energy release rate criterion is used. For a body with a macroscopic crack, the strain energy release rate $G$ is the amount of energy released in advancing the crack by a distance $d a$ normalized by the new crack area. It is typically written as

$$
G=\frac{d W_{s}}{t d a}
$$

where $t$ is the depth of the cracked body (thus, $t d a$ is the new crack area). In adapting the concept of strain energy release rate to continuum damage mechanics, we work with the strain energy density released $W$ rather than the total energy released $W_{s}$ Thus, we use the relation

$$
d W_{s}=V d W
$$

where $V$ is the volume of the material that is being damaged, and the increment in released strain energy density is shown graphically in Fig. 4. By assuming a piecewise linear response, as in Fig. 4, the released strain energy density can be calculated as

$$
d W=\frac{1}{2}\{\sigma(\varepsilon+d \varepsilon)-\varepsilon(\sigma+d \sigma)\}
$$

It is possible to separate the strain energy release rates associated with each tensile damage mode, where again an analogy with a macroscopically cracked body is used. The three modes of loading for such a macroscopic crack are denoted as I, II, and III, and they are associated with opening, in-plane shear, and out-of-plane shear, respectively [26]. Figure 5 illustrates the three modes of loading as they apply to tensile damage in the $x_{i}$ direction, which would be associated with the damage variable $D_{i}^{T}$, where $i, j, k=1,2,3$, and $i \neq j \neq k$. In this scenario, mode I damage is caused by $\sigma_{i i}$ (no

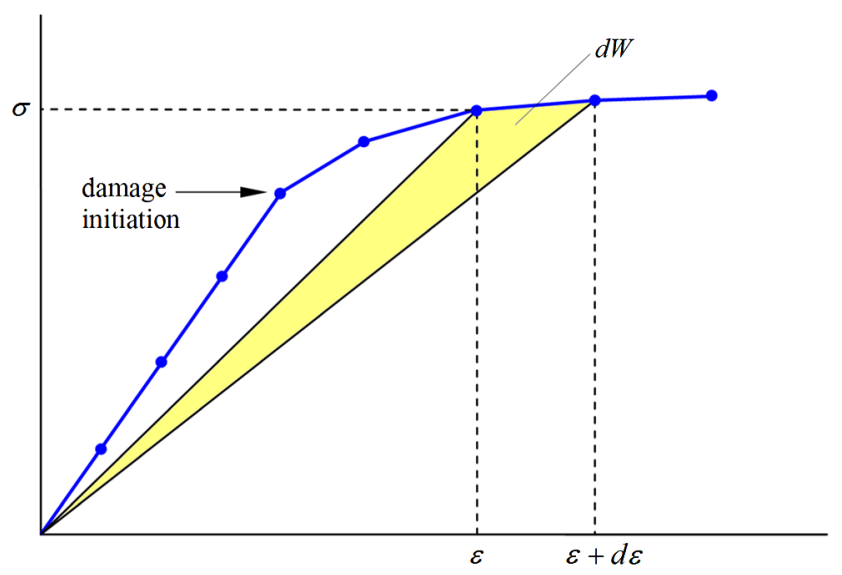

Fig. 4 Graphical representation of the released strain energy density increment.

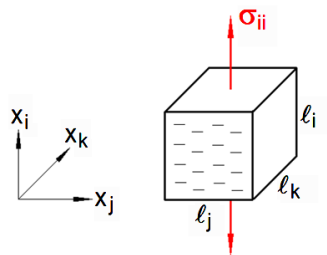

Mode I

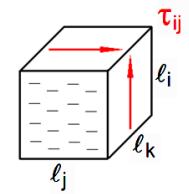

Mode II

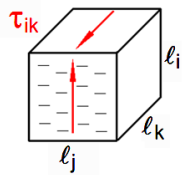

Mode III
Fig. 5 Three loading modes that affect damage in the $x_{i}$ direction.
Table 1 Association between loading modes and stress components for damage associated with the coordinate direction, $x_{i}$

\begin{tabular}{cccccc}
\hline \hline Coordinate direction & & $\begin{array}{c}\text { Stress component associated } \\
\text { with loading mode }\end{array}$ \\
\hline$i$ (damage orientation) & $j$ & $k$ & I & II & III \\
\hline 1 & 2 & 3 & $\sigma_{11}$ & $\tau_{12}$ & $\tau_{13}$ \\
2 & 3 & 1 & $\sigma_{22}$ & $\tau_{23}$ & $\tau_{12}$ \\
3 & 1 & 2 & $\sigma_{33}$ & $\tau_{13}$ & $\tau_{23}$ \\
\hline \hline
\end{tabular}

summation), mode II damage is caused by $\tau_{i j}$, and mode III damage is caused by $\tau_{i k}$ (see Table 1 ). The corresponding mode-specific strain energy density increments are then given by the increments in released energy density, as shown in Fig. 5, associated with the $\sigma_{i i}-\varepsilon_{i i}, \tau_{i j}-\gamma_{i j}$, and $\tau_{i k}-\gamma_{i k}$ response curves.

Because, as indicated in Table 1 , each shear stress component is associated with a loading mode for damage in two directions (i.e., $\tau_{12}$ is associated with mode II loading for damage in the $x_{1}$ direction as well as mode III loading for damage in the $x_{2}$ direction), it is necessary to partition the shear strain energy density increments appropriately. Toward this end, denoting the stress and strain in vector form,

$$
\begin{aligned}
\boldsymbol{\sigma} & =\left[\begin{array}{llllll}
\sigma_{11} & \sigma_{22} & \sigma_{33} & \tau_{23} & \tau_{13} & \tau_{12}
\end{array}\right]^{T} ; \\
\boldsymbol{\varepsilon} & =\left[\begin{array}{llllll}
\varepsilon_{11} & \varepsilon_{22} & \varepsilon_{33} & \gamma_{23} & \gamma_{13} & \gamma_{12}
\end{array}\right]^{T}
\end{aligned}
$$

and denoting the components of the stress and strain vectors as $\sigma_{n}$ and $\varepsilon_{n}$, respectively, with $n=1, \ldots, 6$, the strain energy density release rate associated with each response curve can be denoted as $d W_{n}$. The following partitions can be made according to damage direction in order to arrive at mode-specific strain energy density release rates:

$$
\begin{gathered}
d \hat{W}_{I}^{1}=d W_{1} ; \quad d \hat{W}_{I I}^{1}=\frac{d D_{1}}{d D_{1}+d D_{2}} d W_{6} ; \\
d \hat{W}_{I I I}^{1}=\frac{d D_{1}}{d D_{1}+d D_{3}} d W_{5} ; \quad d \hat{W}_{I}^{2}=d W_{2} ; \\
d \hat{W}_{I I}^{2}=\frac{d D_{2}}{d D_{2}+d D_{3}} d W_{4} ; \quad d \hat{W}_{I I}^{2}=\frac{d D_{2}}{d D_{1}+d D_{2}} d W_{6} ; \\
d \hat{W}_{I}^{3}=d W_{3} ; \quad d \hat{W}_{I I}^{3}=\frac{d D_{3}}{d D_{1}+d D_{3}} d W_{5} ; \\
d \hat{W}_{I I I}^{3}=\frac{d D_{3}}{d D_{2}+d D_{3}} d W_{4}
\end{gathered}
$$

where $d \hat{W}_{M}^{i}$ is the mode-specific increment in released strain energy density for damage in the $x_{i}$ direction and $M$ is the mode (I, II, or III).

The mode-specific strain energy release rates associated with damage in the $x_{i}$ direction can be found using Eqs. (21), (22), and (25) and are given by

$$
G_{M}^{i}=\frac{d \hat{W}_{M}^{i}}{l_{k} d \bar{a}_{M}^{i}} V=\frac{d \hat{W}_{M}^{i}}{l_{k} d \bar{a}_{M}^{i}} l_{i} l_{j} l_{k}=\frac{d \hat{W}_{M}^{i}}{d \bar{a}_{M}^{i}} l_{i} l_{j}
$$

where, as shown in Fig. 5, the lengths of the material in the three directions are denoted by $\bar{l}_{i}, l_{j}$, and $l_{k}$, and $d \bar{a}_{M}^{i}$ is a mode-specific increment in effective crack length, which can be calculated by scaling the length of the material in the damage direction by the increment in stiffness reduction due to the damage variable increment. That is,

$\begin{array}{lll}d \bar{a}_{I}^{1}=l_{2} b_{11}^{T} d D_{1} ; & d \bar{a}_{I I}^{1}=l_{2} b_{61} d D_{1} ; & d \bar{a}_{I I I}^{1}=l_{2} b_{51} d D_{1} ; \\ d \bar{a}_{I}^{2}=l_{3} b_{22}^{T} d D_{2} ; & d \bar{a}_{I I}^{2}=l_{3} b_{62} d D_{2} ; & d \bar{a}_{I I I}^{2}=l_{3} b_{42} d D_{2} ; \\ d \bar{a}_{I}^{3}=l_{1} b_{33}^{T} d D_{3} ; & d \bar{a}_{I I}^{3}=l_{1} b_{43} d D_{3} ; & d \bar{a}_{I I I}^{3}=l_{1} b_{53} d D_{3}\end{array}$

Substituting Eq. (27) into Eq. (26) yields 


$$
\begin{array}{rlrl}
G_{I}^{1} & =\frac{l_{1}}{b_{11}^{T}} \frac{\mathrm{d} \hat{W}_{I}^{1}}{\mathrm{~d} D_{1}} ; & G_{I I}^{1}=\frac{l_{1}}{b_{61}} \frac{d \hat{W}_{I I}^{1}}{d D_{1}} ; & G_{I I I}^{1}=\frac{l_{1}}{b_{51}} \frac{\mathrm{d} \hat{W}_{I I I}^{1}}{\mathrm{~d} D_{1}} ; \\
G_{I}^{2}=\frac{l_{2}}{b_{22}^{T}} \frac{d \hat{W}_{I}^{2}}{d D_{2}} ; & G_{I I}^{2}=\frac{l_{2}}{b_{42}} \frac{\mathrm{d} \hat{W}_{I I}^{2}}{\mathrm{~d} D_{2}} ; & G_{I I I}^{2}=\frac{l_{2}}{b_{62}} \frac{d \hat{W}_{I I I}^{2}}{d D_{2}} ; \\
G_{I}^{3}=\frac{l_{3}}{b_{33}^{T}} \frac{\mathrm{d} \hat{W}_{I}^{3}}{\mathrm{~d} D_{3}} ; & G_{I I}^{3}=\frac{l_{3}}{b_{53}} \frac{d \hat{W}_{I I}^{3}}{d D_{3}} ; & G_{I I I}^{3}=\frac{l_{3}}{b_{43}} \frac{\mathrm{d} \hat{W}_{I I I}^{3}}{\mathrm{~d} D_{3}}
\end{array}
$$

which enables the calculation of the instantaneous mode-specific strain energy release rate for each damage direction (indicated by the superscript).

The strain energy release rates from Eq. (28) can then be used in final-failure criteria by comparing them to the material critical strain energy release rates. For example, one can use the maximum-strain energy release rate criterionm in which final failure occurs when any of the following are satisfied:

$$
G_{M}^{i}=G_{M}^{C}
$$

where $G_{M}^{C}$ is the mode-specific critical strain energy release rate. Alternatively, the power-law criterion is given by

$$
\left(\frac{G_{I}^{i}}{G_{I}^{C}}\right)^{\alpha}+\left(\frac{G_{I I}^{i}}{G_{I I}^{C}}\right)^{\alpha}+\left(\frac{G_{I I I}^{i}}{G_{I I I}^{C}}\right)^{\alpha}=1
$$

where $\alpha$ is a material parameter. Finally, the Benzeggagh-Kenane (B-K) [27] criterion can be employed, which is applicable only when $G_{I I}^{C}=\overline{G_{I I I}^{C}}$. The B-K criterion can be expressed as

$$
G_{I}^{i}+G_{I I}^{i}+G_{I I I}^{i}=G^{C}
$$

with

$$
G^{C}=G_{I}^{C}+\left(G_{I I}^{C}-G_{I}^{C}\right)\left(\frac{G_{I I}^{i}+G_{I I I}^{i}}{G_{I}^{i}+G_{I I}^{i}+G_{I I I}^{i}}\right)^{\eta}
$$

where $\eta$ is a material parameter.

In compression, the failure mechanism is assumed not to be related to cracking, and thus a strain energy release rate based final-failure criterion is not applicable. Instead, a criterion based on the total dissipated energy associated with a damage direction is employed. This criterion can be expressed as

$$
\left(\hat{W}_{I}^{i}+\hat{W}_{I I}^{i}+\hat{W}_{I I I}^{i}\right) V=W_{s}^{C}
$$

where $W_{s}^{C}$ is a critical strain energy and $\hat{W}_{M}^{i}$ is the mode-specific strain energy release rate, which can be determined by integrating the mode-specific strain energy release rate increments.

\section{Results and Discussion}

To demonstrate the capabilities of the HFGMC micromechanics model, including the proposed progressive damage model, a commonly modeled composite system has been chosen. This is the $E$-glass/MY750/HY917/DY063 epoxy resin composite that was included in the WWFE [1]. As is often the case, while nominal constituent (fiber/matrix) elastic and strength properties were provided in the WWFE, nonlinear stress-strain curves of the constituents, as are desirable for nonlinear micromechanics model input, were not provided. As such, the constituent properties were obtained based on correlation with composite ply-level nonlinear data provided in the WWFE for the $E$-glass/epoxy composite (i.e., the constituent properties were backed out).

All of the results presented consider a $7 \times 7$ subcell RUC with a fiber volume fraction of 0.6, as shown in Fig. 6. While HFGMC is mesh-dependent, this HFGMC unit cell discretization was shown to yield a converged global nonlinear deformation response for a $\mathrm{SiC} / \mathrm{Ti}$ composite by Bednarcyk et al. [28] Note that the doubly periodic RUC shown is infinitely long in the $x_{1}$ direction and, thus, represents a special case of the triply periodic version of HFGMC described previously. It should also be noted that, for nonlinear analysis, HFGMC tracks the local fields within each subcell at a number of

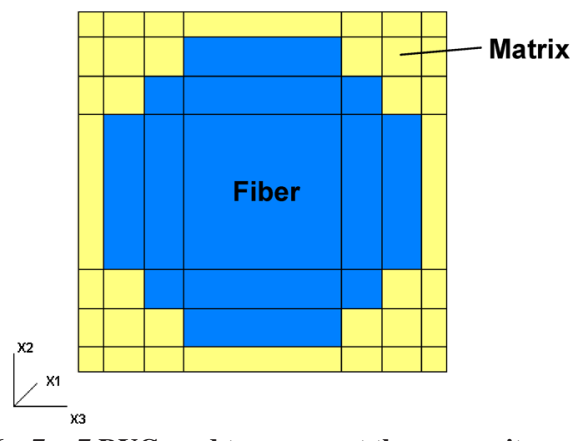

Fig. $67 \times 7$ RUC used to represent the composite material.

integration points. Three integration points were used for this purpose in both the $x_{2}$ and $x_{3}$ directions, for a total of nine integration points per subcell. Obviously, damage will initiate and evolve differently at each of these nine points per subcell; thus, an average of the damage at these nine points was used to determine the subcell damaged elastic properties.

The simulations reported next were executed on a standard dualcore Windows workstation. The execution times for generating the stress-strain response of the neat epoxy was a fraction of a second ( $\sim 0.1 \mathrm{~s})$, whereas, the stress-strain curves for the composite (using the $7 \times 7$ unit cell) required approximately $3 \mathrm{~s}$ of execution time. Generation of the composite failure envelopes was accomplished by repeatedly simulating the stress-strain response along radial paths with a given angular increment in the given biaxial stress space under stress control. That is, to generate a simulated failure envelope in $\sigma_{11}-\sigma_{22}$ stress space, a simulation was first run by monotonically increasing $\sigma_{11}$ with all other stress components equal to zero until final failure was predicted. Then, a biaxial stress-strain simulation (monotonically increasing both $\sigma_{11}$ and $\sigma_{22}$ simultaneously) was run, where $\tan ^{-1}\left(\sigma_{22} / \sigma_{11}\right)=5^{\circ}$ (for example) until final failure. This process continued until the entire envelope was generated through the complete $360^{\circ}$ in the stress space. Because the failure envelope generation is automated within the HFGMC code, these composite failure envelope simulations typically require only several minutes ( $\sim 4 \mathrm{~min}$ ) of execution time.

Figures $7 \mathrm{a}-7 \mathrm{c}$ show the correlation of the micromechanics model with the composite ply-level experimental data, provided by Hinton et al. [1] , used to determine the constituent properties. The progressive damage model assumes that all material nonlinearity is due to stiffness reduction rather than inelastic deformation. Unloading behavior of the composite, were it available, could be examined to assess the validity of this assumption. The determined constituent data are summarized in Tables $\underline{2}$ and $\underline{3}$. While the resin matrix is subject to the proposed progressive damage model, the fiber is treated as isotropic and linear elastic until failure. A maximum stress criterion is used for the fiber, based only on tensile and compressive longitudinal stresses. Once the fiber fails, all of its elastic stiffness properties are instantaneously reduced to a very low value (i.e., $D_{i}^{\bullet}$ are set to 0.9999 , see Eqs. (1-4)). It is noted that the fiber strengths determined via correlation in Table 2 are slightly lower than the nominal values of 2150 and $1450 \mathrm{MPa}$ given by Hinton et al. [1] Further, in the model longitudinal composite tensile response, shown in Fig. 7a, it is noted that some nonlinearity due to matrix damage is present before failure.

As stated, the matrix is assumed to be subject to the proposed progressive damage model. An exponential form was assumed for the instantaneous modified slope [see Eq. (20)] of the matrix material stress-strain curve after damage initiation:

$$
k_{i}^{\prime}=A e^{-\varepsilon_{i}^{D} / B}
$$

where the constants $A$ and $B$ are permitted to take on different values in tension $T$ and compression $C$. As indicated in Table 3 , these constants were determined via correlation with the ply-level nonlinear composite response curves provided by Hinton et al. [1] For final failure of a subcell in tension, the maximum-strain energy 


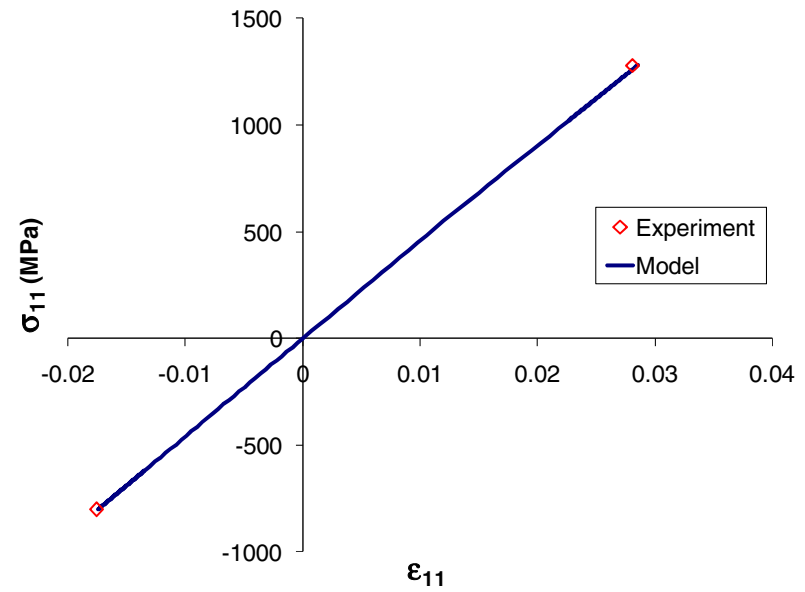

a)

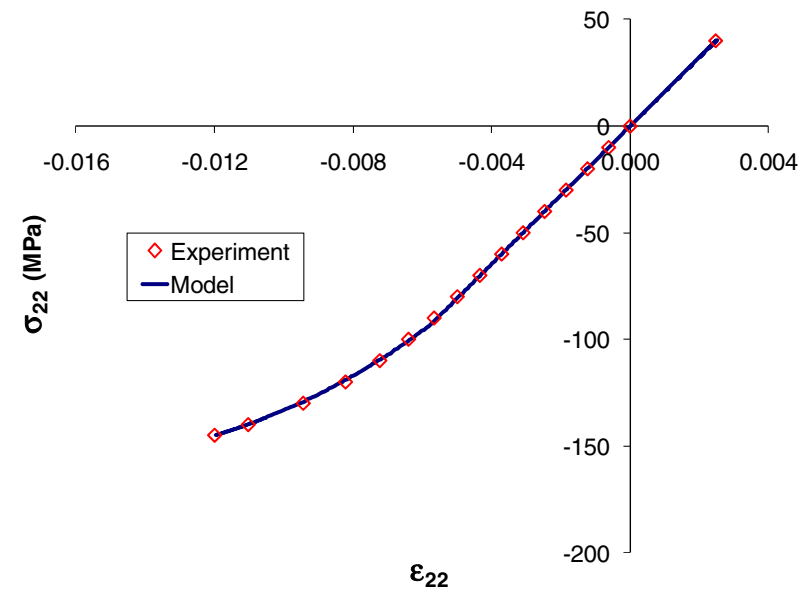

b)

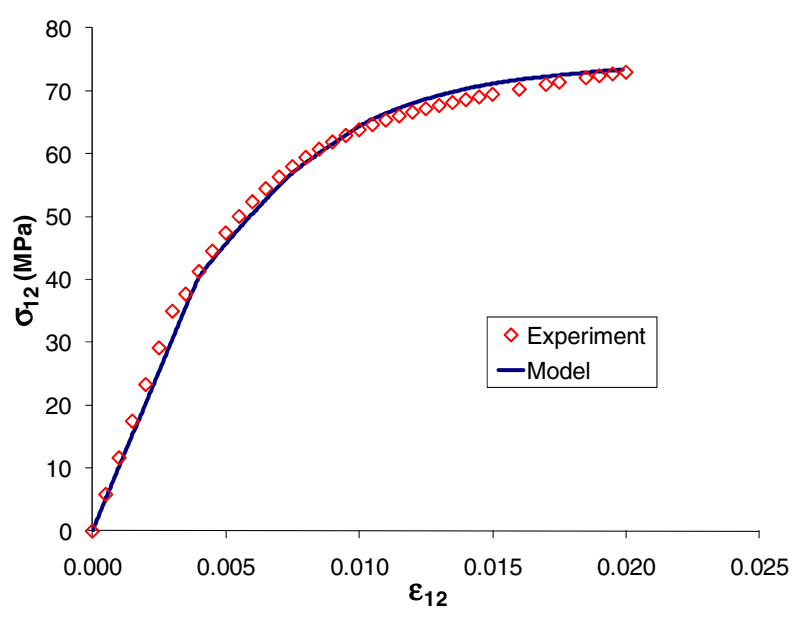

c)

Fig. 7 Model correlation with experiment for a) the longitudinal tensile and compressive response, b) transverse tensile and compressive response, and c) the longitudinal shear response of $E$-glass/epoxy.

Table 2 Properties of Silenka $E$-glass fiber

\begin{tabular}{lcclc}
\hline \hline Property & Symbol & Units & \multicolumn{1}{c}{ Source } & Value \\
\hline Elastic modulus & $E$ & $\mathrm{GPa}$ & Hinton et al. [1] tabular value & 74 \\
Poisson's ratio & $v$ & $\overline{\mathrm{MPa}}$ & Hinton et al. [1] t tabular value & 0.2 \\
Longitudinal tensile strength & $X^{T}$ & $\mathrm{MPr}$ & Corration (Fig. 7a) & 2110 \\
Longitudinal compressive strength & $X^{C}$ & $\mathrm{MPa}$ & Correlation (Fig. $\underline{7 \mathrm{a}}$ ) & 1290 \\
\hline \hline
\end{tabular}

Table 3 Properties of MY750/HY917/DY063 epoxy

\begin{tabular}{|c|c|c|c|c|}
\hline Property & Symbol & Units & Source & Value \\
\hline Elastic modulus & $E$ & GPa & Correlation (Fig. 7b) & 3.7 \\
\hline Poisson's ratio & $v$ & - & Hinton et al. [1] tabular value & 0.35 \\
\hline Tensile damage initiation strain & $X_{\varepsilon}^{T}$ & - & Correlation (Fig. 7b) & 0.0125 \\
\hline Compressive damage initiation strain & $X_{\varepsilon}^{C}$ & - & Correlation (Fig. $\underline{7 b}$ ) & 0.0287 \\
\hline Engineering shear damage initiation strain & $S_{\varepsilon}$ & 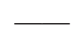 & Correlation (Fig. $\overline{7 \mathrm{c}}$ ) & 0.0443 \\
\hline Postdamage slope parameter, Eq. (34) & $A^{\underline{E}}$ & - & Correlation (Fig. $\overline{7 \mathrm{c}}$ ) & 0.7 \\
\hline Postdamage slope parameter, Eq. ( $\overline{34})$ & $A^{C}$ & 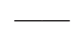 & Correlation (Fig. $\overline{7 b}$ ) & 2.0 \\
\hline Postdamage slope parameter, Eq. ( $\overline{34})$ & $B^{T}$ & $\longrightarrow$ & Correlation (Fig. $\overline{7 \mathrm{c}}$ ) & 0.82 \\
\hline Postdamage slope parameter, Eq. $(\overline{34})$ & $B^{C}$ & 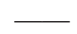 & Correlation (Fig. $\overline{7 b}$ ) & 0.96 \\
\hline Tensile scaling parameter, Eq. (2) & $b_{i i}^{T}$ & $\longrightarrow$ & Correlation (Figs. $\overline{7 b}$ and $7 \mathrm{c}$ ) & 1.32 \\
\hline Compressive scaling parameter, Eq. (2) & $b_{i i}^{C}$ & 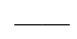 & Correlation (Figs. $\overline{\overline{7 b}}$ and $\overline{\overline{7 c}}$ ) & 1.32 \\
\hline Shear scaling parameters, Eq. (3) & $b_{4 i}, b_{5 i}, b_{6 i}$ & & Default value - & 0.5 \\
\hline Mode I critical strain energy release rate & $G_{I}^{C}$ & $\mathrm{~J} / \mathrm{m}^{2}$ & Correlation (Fig. $7 \mathrm{~b}$ ) & 800 \\
\hline Modes II and III critical strain energy release rates & $G_{I I}^{C}\left(=G_{I I I}^{C}\right)$ & $\mathrm{J} / \mathrm{m}^{2}$ & Correlation (Fig. $\overline{7 \mathrm{c}}$ ) & 2400 \\
\hline Critical compressive strain energy & $W_{s}^{C}$ & $\mathrm{~J}$ & Correlation (Fig. $\overline{7 b})$ & $1.86 \times 10^{-6}$ \\
\hline Material length & $l_{i}$ & $\mathrm{~m}$ & Correlation (Figs. $\overline{7 b}$ and $\underline{7 c}$ ) & $9 \times 10^{-5}$ \\
\hline
\end{tabular}


release rate criterion [Eq. (29)] was used. It should also be noted, in Table 3, that the elastic modulus value employed for the matrix is higher than the 3.35 GPa nominal value provided by Hinton et al. [1] Further, the tensile and compressive scaling parameters $\left(b_{i i}^{T}, b_{i i}^{\bar{C}}\right)$ were chosen to scale the effect of normal damage with respect to shear damage. The default shear scaling parameters $\left(b_{4 i}, b_{5 i}, b_{6 i}\right)$ were employed. These parameters provide the damage model with the capability to behave differently in shear vs normal deformation, despite the fact that identical $A$ and $B$ parameters control the damage evolution in both cases. Hence, one can correlate the normal response using default scaling parameters and then choose the shear scaling parameters to enable capture of the shear response, or vice versa (as done here). As the damage model is presented herein, the scaling parameters can also be assigned different values per stiffness component, but this would result in anisotropic progressive damage behavior of an initially undamaged material and is thus not desirable in the present application to the neat resin matrix. Finally [from Eqs. (28) and (33)] it is clear that the critical strain energy release rates and the critical compressive strain energy are scaled by the material lengths. Therefore, these parameters are not independent and, by choosing a different material length, different values of the critical strain energy release rates and critical compressive strain energy would result from the correlation with the ply-level composite data.

Figure 8 shows the monolithic resin matrix behavior that results from the parameters given in Table 3 . Most noteworthy is the extremely brittle behavior in tension compared with the ductileappearing behavior in compression and shear. This brittle behavior is a direct result of the brittle transverse tensile composite behavior observed in Fig. 7b that was used to characterize the matrix tensile damage/failure response. Obviously, it would not be possible to capture these vast differences in the character of the damage/failure behavior among tension, compression, and shear via a simple failure criterion that treats the epoxy as linear to failure, as shown in [6]. Similarly, a nonlinear model similar to plasticity, which characterizes nonlinearity through a single scalar measure (i.e., von Mises stress), would miss the brittle character in tension if it were to capture the more ductile-appearing character in compression and shear. Thus, in the context of micromechanics, the need for a damage model that can handle material nonlinearity and also separate the damage modes is clear, as is offered by the present model.

Figures 9-11 provide the model damage initiation and final-failure envelopes for the monolithic (neat) epoxy resin, given the material properties in Table 3 . The distance between the two envelopes in each figure indicates the degree to which the predicted failure is progressive vs sudden. For instance, in Fig. 9, it is clear that, in the tension-tension regime (quadrant I), the failure is very brittle, with virtually no progression of damage between initiation and final failure. In the compression-compression regime (quadrant III), however, there is a great deal of progression before final failure.

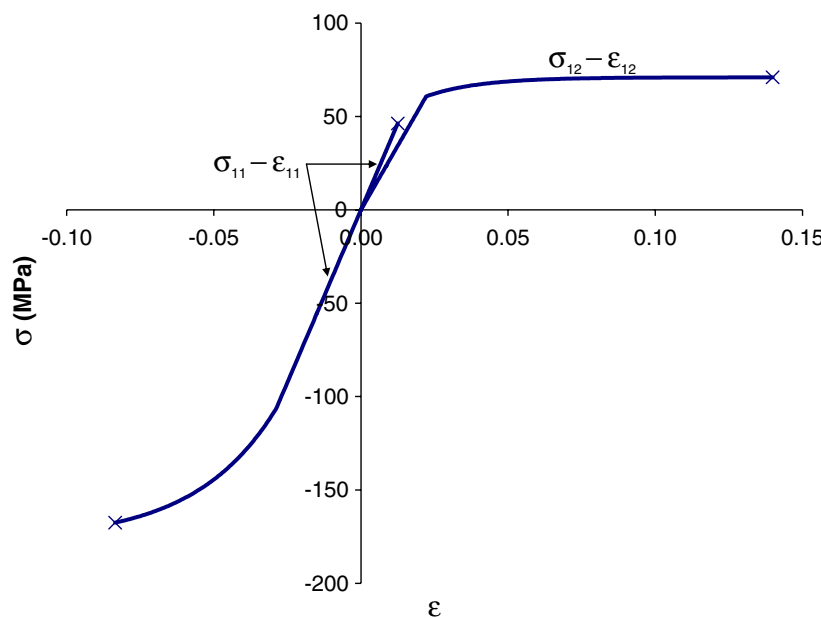

Fig. 8 Monolithic (neat) epoxy resin behavior simulated via the proposed damage model, given the material properties shown in Table $\underline{3}$.

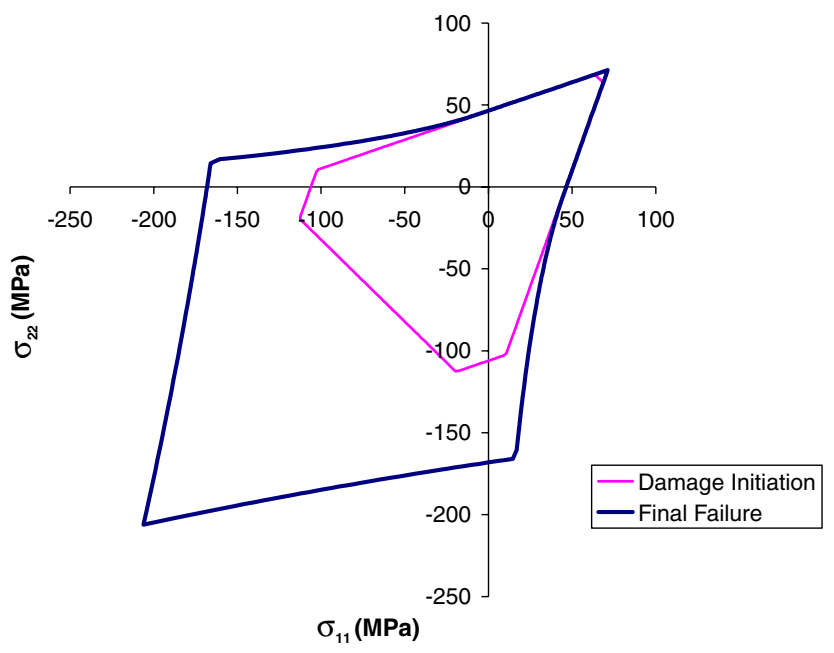

Fig. 9 Model damage initiation and final-failure envelopes for the monolithic (neat) epoxy in the biaxial normal stress plane.

Figure 10 provides the normal-shear failure envelopes for the case in which the normal and shear components are interactive. That is, examining Eq. (10), it is clear that the $\varepsilon_{11}$ and $\varepsilon_{12}$ strain components are interactive, in that they both contribute to the damage strain $\varepsilon_{1}^{D}$. This interaction between the 11-normal and 12-shear behavior is manifested as the elliptical character of the surfaces in Fig. 10. Figure 11, on the other hand, provides the normal-shear envelopes for the case in which the components do not interact. Again examining Eq. (10), it is clear that $\varepsilon_{11}$ contributes only to damage strain $\varepsilon_{1}^{D}$, while $\varepsilon_{23}$ contributes to $\varepsilon_{2}^{D}$ and $\varepsilon_{3}^{D}$; thus, these components do not interact. The result is the more rectangular character of the surfaces in Fig. 11. Thus, the material, which was initially isotropic, is highly anisotropic in terms of its multiaxial damage progression and failure response. It is noted that the damage surfaces in Fig. 11 do display some interaction effects because the surfaces are generated in stress space, so application of $\sigma_{11}$ with $\sigma_{22}=0$ results in nonzero $\varepsilon_{22}$ and $\varepsilon_{33}$ due to Poisson effects.

Another important feature of the implementation of the proposed progressive damage model is illustrated in Fig. 10. When the normal stress component transitions from tensile to compressive (quadrant I to quadrant II transition), there is no jump in the final-failure envelope as one might expect (as the compressive damage

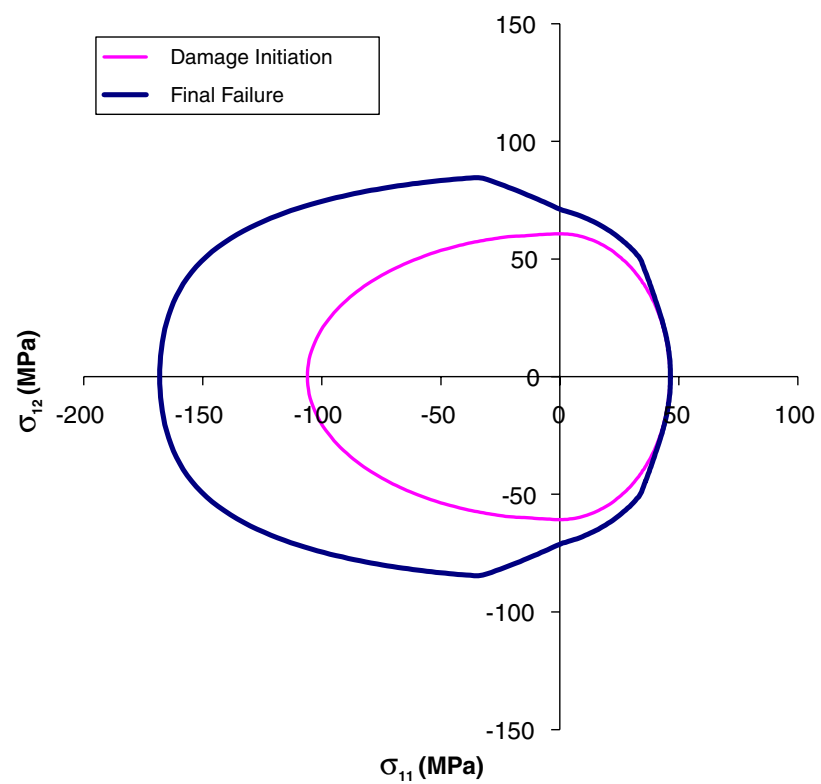

Fig. 10 Model damage initiation and final-failure envelopes for the monolithic (neat) epoxy in the normal-shear stress plane with interaction. 


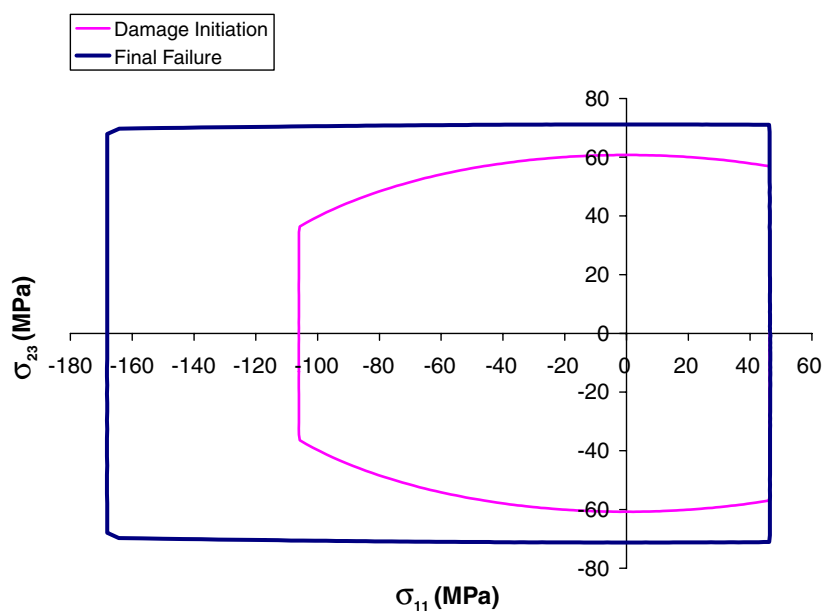

Fig. 11 Model damage initiation and final-failure envelopes for the monolithic (neat) epoxy in the normal-shear stress plane without interaction.

parameters in Table $\underline{3}$ become active). Rather, the transition is gradual. This feature was incorporated by basing the transition in properties on the degree to which the damage strain component is shear dominated. That is, when a damage strain is completely shear dominated (i.e., on the $y$ axis of Fig. 10), the tensile parameters are used. If the stress state is compressive, the parameters are interpolated between the tensile and compressive values until the damage strain is no longer dominated by shear, at which point they become the compressive values.

Figures 12-14 show model composite damage initiation and finalfailure envelopes. The experimental data are taken from Hinton et al. [1] The experimental data on the axes are the data used to characterize the damage model for the matrix material. The lack of agreement in quadrant IV indicates that a different final-failure criterion, such as the power-law or B-K criteria, should be employed. The interaction of strain energy release rates provided by these criteria may improve the agreement. In Fig. 14, the noncharacterization experimental data are actually for a $0 . \overline{60}$ volume fraction $E$-glass/LY556/HT907/ DY063 epoxy composite [1], for which the properties are similar to the $E$-glass/MY750/HY917/DY063 epoxy composite, for which the materials employed in the simulation were characterized. As shown, the agreement is quite good, most notably in the ability of the model to capture the observed strengthening in quadrant II.

As a final example, the progressive damage model has been characterized based on the in situ matrix shear response measured and presented by $\mathrm{Ng}$ et al. [29] The specific type of fiber and composition of the polymer matrix were not disclosed by these authors for proprietary reasons. Using a novel technique, $\mathrm{Ng}$ et al. [29] have determined the average nonlinear in-plane shear behavior

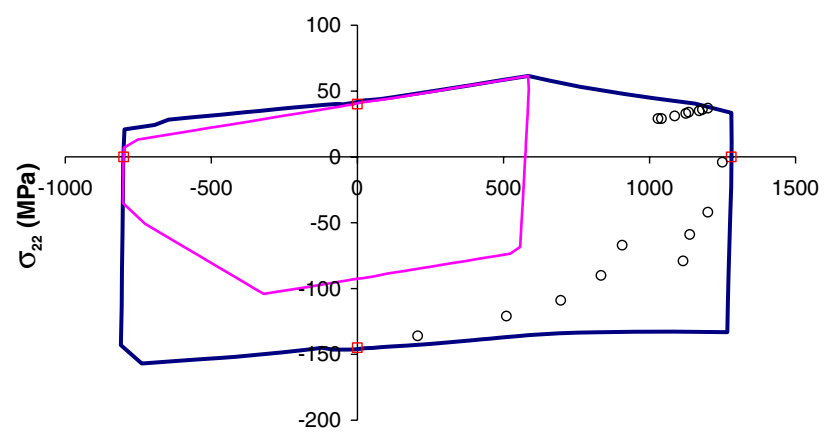

\begin{tabular}{l} 
- Final Failure \\
- Damage Initiation \\
$\square$ Experiment (Characterization) \\
$\circ$ Experiment \\
\hline
\end{tabular}

$\sigma_{11}(\mathrm{MPa})$

Fig. 12 Model damage initiation and final-failure envelopes for unidirectional $\boldsymbol{E}$-glass/epoxy in the biaxial normal in-plane stress plane.

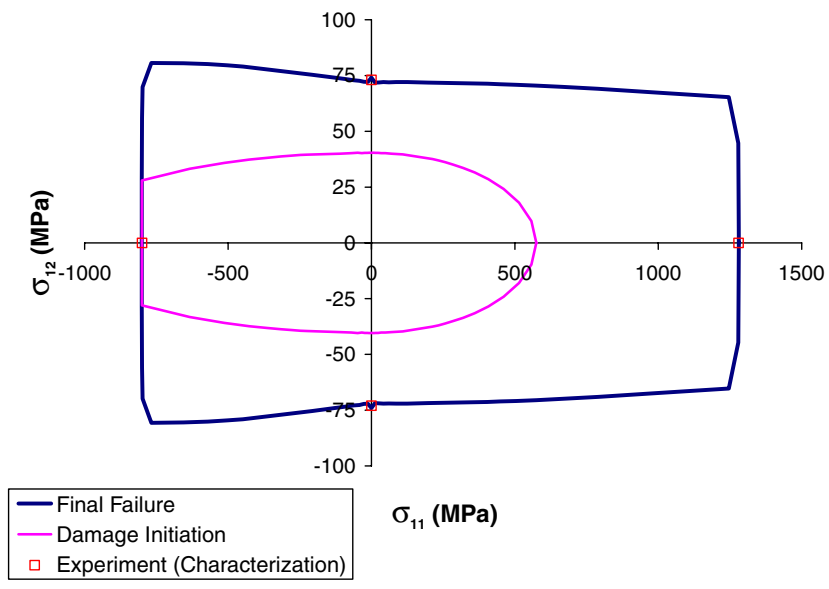

Fig. 13 Model damage initiation and final-failure envelopes for unidirectional $E$-glass/epoxy in the longitudinal tension: in-plane shear stress plane.

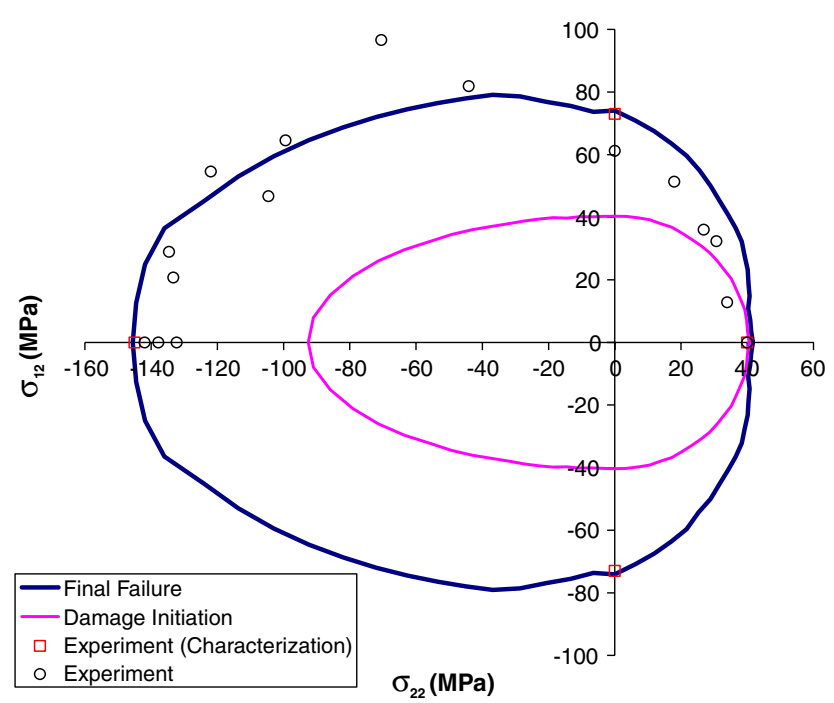

Fig. 14 Model damage initiation and final-failure envelopes for unidirectional $E$-glass/epoxy in the transverse tension: in-plane shear stress plane.

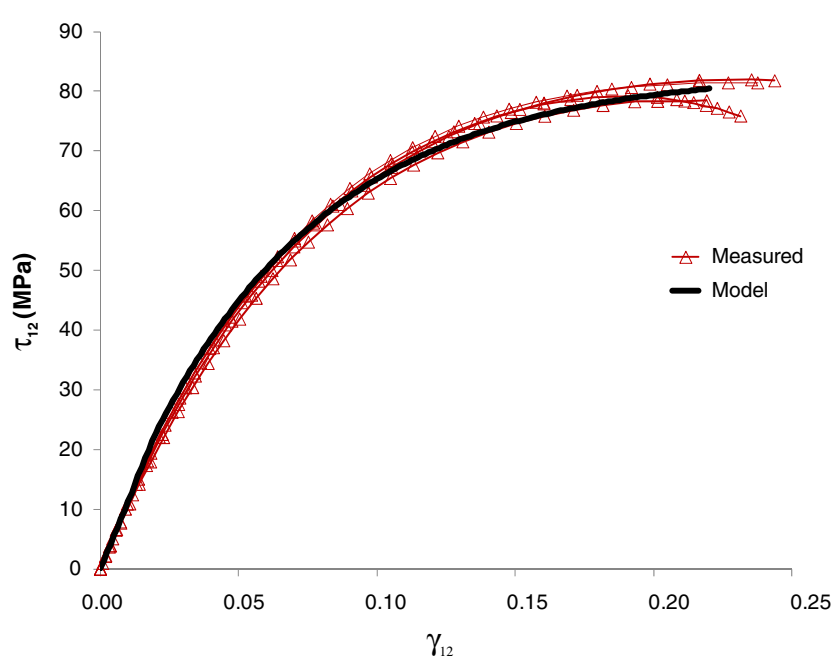

Fig. 15 Model correlation with polymer matrix shear response measured by $\mathrm{Ng}$ et al. [29]. 
Table 4 Properties of the composite constituents considered by Ng et al. [29]

\begin{tabular}{|c|c|c|c|c|}
\hline Property & Symbol & Units & Source & Value \\
\hline Fiber axial elastic modulus & $E_{11}$ & $\mathrm{GPa}$ & $\mathrm{Ng}$ et al. [29] tabular value & 310 \\
\hline Fiber transverse elastic modulus & $E_{22}$ & $\mathrm{GPa}$ & $\mathrm{Ng}$ et al. [29] tabular value & 20.5 \\
\hline Fiber major Poisson’s Ratio & $v_{12}$ & & $\mathrm{Ng}$ et al. [29] tabular value & 0.28 \\
\hline Fiber minor Poisson's Ratio & $v_{23}$ & & $\mathrm{Ng}$ et al. [29] tabular value & 0.35 \\
\hline Fiber axial shear modulus & $G_{12}$ & $\mathrm{GPa}$ & $\mathrm{Ng}$ et al. [29] tabular value & 32.8 \\
\hline Matrix elastic modulus & $E$ & $\mathrm{GPa}$ & $\mathrm{Ng}$ et al. $[\overline{29}]$ & 3.078 \\
\hline Matrix Poisson's ratio & $v$ & & $\mathrm{Ng}$ et al. $[\overline{29}]$ & 0.35 \\
\hline Matrix engineering shear damage initiation strain & $S_{\varepsilon}$ & & Correlation (Fig. 15) & 0.02 \\
\hline Matrix postdamage slope parameter, Eq. (34) & $A^{T}$ & 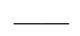 & Correlation (Fig. 15 ) & 1.1 \\
\hline Matrix postdamage slope parameter, Eq. $(\overline{34})$ & $B^{T}$ & & Correlation (Fig. $\overline{15}$ ) & 3.3 \\
\hline Matrix shear scaling parameters, Eq. (3) & $b_{4 i}, b_{5 i}, b_{6 i}$ & & Default value - & 0.5 \\
\hline $\begin{array}{l}\text { Matrix modes II and III critical strain energy } \\
\text { release rates }\end{array}$ & $G_{I I}^{C}\left(=G_{I I I}^{C}\right)$ & $\mathrm{J} / \mathrm{m}^{2}$ & Correlation (Fig. 15) & 1240 \\
\hline Matrix material length & $l_{i}$ & $\mathrm{~m}$ & Correlation (Fig. 15) & $9 \times 10^{-5}$ \\
\hline
\end{tabular}

of the composite at the ply level, as well as that of the matrix. Using the four matrix stress-strain curves given by these authors, the correlation of the progressive damage model with the measured data is presented in Fig. 15. The corresponding material parameters for the polymer matrix are given in Table 4 , along with the fiber material properties provided by $\mathrm{Ng}$ et al. [29]. It is evident from Fig. 15 that the simple exponential form of the instantaneous slope given in Eq. (34) captures the general character of the matrix nonlinear shear response reasonably well, but it is unable to precisely capture the early stiffness decrease of the matrix as well as the saturation behavior close to failure. A different functional form for the instantaneous stiffness could provide more precise correlation in this regard. Note that the failure point for the model was chosen as the average ultimate shear strength of the four measured shear curves (80.4 MPa). This point corresponded to a mode II/III strain energy release rate of $1240 \mathrm{~J} / \mathrm{m}^{2}$. Note also that it was assumed for both the matrix and the composite that the only contribution to failure was matrix shear damage and failure; thus, some model data that were given in the previous examples are not needed in the present simulations.

A comparison of the predicted composite in-plane shear response with the data provided by $\mathrm{Ng}$ et al. [29] is given in Fig. 16. As before, the HFGMC RUC shown in Fig. 6 was used, with nine integration points per subcell. As was the case for the correlated matrix shear response (Fig. 15), the model does a reasonably good job of predicting the composite response. The slight overprediction of the measured curves near damage initiation and the inability to capture well the saturation behavior is also similar to what was observed in the model characterization for the matrix. The failure prediction for the composite was taken as the point at which the average matrix strain energy release rate reached $1240 \mathrm{~J} / \mathrm{m}^{2}$. This approach was

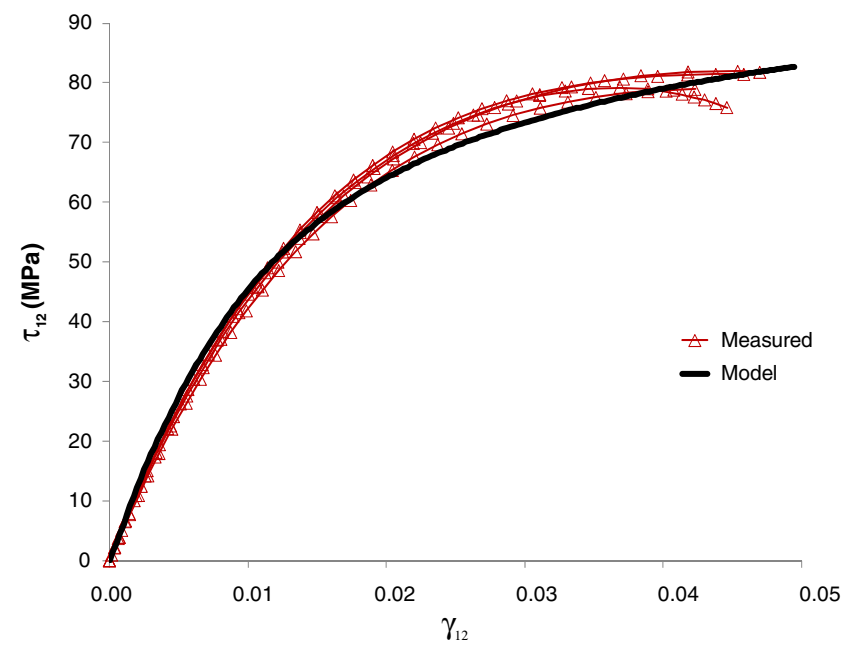

Fig. 16 Comparison of the predicted $V f=68 \%$ polymer matrix composite in-plane shear response with that measured by $\mathrm{Ng}$ et al. [29]. used because the critical strain energy release rate was determined from the matrix shear response curves given by $\mathrm{Ng}$ et al. [29], which provide the average in situ matrix behavior. If the failure prediction was based on failure of the first subcell, it would have occurred at a very low stress-and-strain value $\left(\tau_{12}=65.3 \mathrm{MPa}, \gamma_{12}=0.021\right)$, along the same model curve shown in Fig. 16. The average matrix strain energy release rate approach overpredicted both the ultimate shear strength and shear strain to failure of the composite. However, this may be due to the fact that the matrix and composite saturation behavior have not been well captured by the exponential form for the instantaneous slope used in the simulations. Aspects of the techniques employed by $\mathrm{Ng}$ et al. [29] to determine the matrix shear response could contribute to some of the discrepancy as well.

\section{Conclusions}

The HFGMC micromechanics model has been extended to include progressive damage at the constituent (subcell) level. The proposed coupled micromechanics-damage model seeks to include multiaxiality, such that the character of the constituent behavior can be vastly different in tension, compression, and shear in as simple a way as possible. It appears that enabling this distinct behavior based on type of loading is beneficial to predicting the damage and failure behavior of polymer matrix composites. The proposed damage model attributes all material nonlinearity to stiffness reduction and is thus less applicable to materials that exhibit plastic deformation. The model's true applicability to a given material can be assessed by examining the material's unloading response to determine the proportion of the nonlinear deformation that is permanent. The damage model introduces six damage parameters (three for tension and three for compression) and associated damage strains based on oriented continuum damage within the constituent materials. Assuming that the damage strains adequately capture the multiaxial nature of the damage, evolution equations for the damage parameters can be established based on the postdamage initiation shape of the constituent stress-strain response. Final failure of a constituent subcell has also been addressed by calculating mode-specific strain energy release rates in tension and a total dissipated strain energy in compression. The coupled HFGMC-damage model has been characterized for an E-glass/epoxy composite based on data in the literature that exhibit brittle behavior in transverse tension but ductile-appearing (i.e., nonlinear) behavior in transverse compression and longitudinal shear. It was shown that the HFGMC-damage model can capture this behavior, and neat resin and composite-level stress-strain curves, damage initiation envelopes, and final-failure envelopes were presented for this material system. A final example was given in which the HFGMC-damage model was applied to predict the shear response of a composite for which the matrix shear response was available in the literature. Future investigation of the presented model will focus on extension to multiscale analysis of resin matrix composite laminates and structures, the latter of which will necessitate linkage of the couple micromechanics-damage model with a finite-element solver. 


\section{References}

[1] Hinton, M. J., Kaddour, A. S., and Soden, P. D. (eds.) Failure Criteria in Fiber Reinforced Polymer Composites: The World-Wide Failure Exercise, Elsevier, New York, 2004

[2] Bogetti, T. A., Hoppel, C. P. R., Harik, V. M., Newill, J. F., and Burns, B. P., "Predicting the Nonlinear Response and Failure of Composite Laminates: Correlation with Experimental Results," Composites Science and Technology, Vol. 64, Nos. 3-4, 2004, pp. 477-485. doi:10.1016/S0266-3538(03)00223-9

[3] Nelson, E. E., Hanson, A. C., Welsh, J. S., and Mayes, J. S., "Recent Advances in Failure Predictions of Composite Laminates Utilizing Multicontinuum Technology," Proceedings of the 49th AIAA/ASME/ ASCE/AHS/ASC Structures, Structural Dynamics, and Materials Conference, AIAA Paper 2008-2319, April 2008.

[4] Aboudi, J., "Micromechanical Analysis of Fully Coupled ElectroMagneto-Thermo-Elastic Multiphase Composites," Smart Materials and Structures, Vol. 10, No. 5, 2001, pp. 867-877. doi:10.1088/0964-1726/10/5/303

[5] Aboudi, J., "The Generalized Method of Cells and High-Fidelity Generalized Method of Cells Micromechanical Models: A Review," Mech. Adv. Materl. Struct., Vol. 11, Nos. 4--5, 2004, pp. 329-366. doi: $10.1080 / 15376490490451543$

[6] Moncada, A. M., Chattopadhyay, A., Bednarcyk, B. A., and Arnold, S. M., "Micromechanics-Based Progressive Failure Analysis of Composite Laminates Using Different Constituent Failure Theories," Proc. 49th AIAA/ASME/ ASCE/AHS/ASC Structures, Structural Dynamics, and Materials Conference, AIAA Paper 2008-1826, April 2008.

[7] Kachanov, L. M., "On the Creep Fracture Time," Izvestiya Akademii Nauk SSSR, Otdeleniya Tekhnika Nauk, No. 8, No. 1, 1958, pp. 26-31 (in Russian).

[8] Kachanov, L. M., Introduction to Continuum Damage Mechanics, Martinus-Nijhoff, Dordrecht, The Netherlands, 1986.

[9] Lemaitre, J., and Chaboche, J.-L., Mechanics of Solid Materials, Cambridge Univ. Press, Cambridge, England, U.K., 1990.

[10] Krajcinovic, D., Damage Mechanics, Elsevier, New York, 1996.

[11] Voyiadjis, G. Z., and Kattan, P. I., Damage Mechanics, Taylor and Francis, Boca Raton, FL, 2005.

[12] Talreja, R., "A Continuum Mechanics Characterization of Damage in Composite Materials," Proceedings of the Royal Society of London A, Vol. 399, No. 1817, 1985, pp. 195-216. doi:10.1098/rspa.1985.0055

[13] Talreja, R., "Transverse Cracking and Stiffness Reduction in Composite Laminates," Journal of Composite Materials, Vol. 19, No. 4, 1985, pp. 355-375. doi: $10.1177 / 002199838501900404$

[14] Matzenmiller, A., Lubliner, J., and Taylor, R. L., "A Constitutive Model for Anisotropic Damage in Fiber-Composites," Mechanics of Materials, Vol. 20, No. 2, 1995, pp. 125-152. doi:10.1016/0167-6636(94)00053-0

[15] "LS-DYNA Keyword User's Manual Vol. 2: Material Models," Livermore Software Technology Corp., Livermore, CA, 2007.

[16] Schweizerhof, K., Weimar, K., Munz, T., and Rottner, T.,
"Crashworthiness Analysis with Enhanced Composite Material Models in LS-DYNA: Merits and Limits" [online paper], 5th International LS-DYNA World Conference, Livermore Software Technology Corp., Troy, MI, 1998, http://www.dynasupport.com/ howtos/material/composite-models/composite_paper.pdf [retrieved 6 May 2010].

[17] "ABAQUS Analysis User's Manual Volume 3: Materials," Dassault Systèmes Simulia Corp., Providence, RI, 2008.

[18] Hashin, Z., "Failure Criteria for Unidirectional Fiber Composites," Journal of Applied Mechanics, Vol. 47, No. 2, 1980, pp. 329-334. doi:10.1115/1.3153664

[19] Bednarcyk, B. A., and Arnold, S. M., "MAC/GMC 4.0 User's Manual," NASA TM 2002-212007, 2002.

[20] Aboudi, J., Pindera, M.-J., and Arnold, S. M., "High-Fidelity Generalized Method of Cells for Inelastic Periodic Multiphase Materials," NASA TM 2002-211469, 2002.

[21] Aboudi, J., Pindera, M.-J., and Arnold, S. M., "Higher-Order Theory for Periodic Multiphase Materials with Inelastic Phases," Int. J. Plasticity, Vol. 19, 2003, pp. 805-847. doi:10.1016/S0749-6419(02)00007-4

[22] Pindera, M.-J., and Bednarcyk, B. A., "An Efficient Implementation of the Generalized Method of Cells for Unidirectional, Multi-Phased Composites with Complex Microstructures," Composites. Part B, Engineering, Vol. 30, No. 1, 1999, pp. 87-105. doi:10.1016/S1359-8368(98)00040-7

[23] Bansal, Y., and Pindera, M.-J., "Testing the Predictive Capability of the High-Fidelity Generalized Method of Cells Using an Efficient Reformulation," NASA CR 2004-213043, 2004.

[24] Arnold, S. M., Bednarcyk, B. A., and Aboudi, J., "Comparison of the Computational Efficiency of the Original Versus Reformulated HighFidelity Generalized Method of Cells," NASA TM 2004-213438, 2004.

[25] Haj-Ali, R., and Aboudi, J., "Nonlinear Micromechanical Formulation of the High Fidelity Generalized Method of Cells," International Journal of Solids and Structures, Vol. 46, No. 13, 2009, pp. 2577-2592. doi:10.1016/j.ijsolstr.2009.02.004

[26] Anderson, T. L., Fracture Mechanics Fundamentals and Application, CRC Press, Boston, 1991

[27] Benzeggagh, M. L., and Kenane, M., "Measurement of Mixed-Mode Delamination Fracture Toughness of Unidirectional Glass/Epoxy Composites with Mixed-Mode Bending Apparatus," Composites Science and Technology, Vol. 56, No. 4, 1996, pp. 439-449. doi:10.1016/0266-3538(96)00005-X

[28] Bednarcyk, B. A., Arnold, S. M., Aboudi, J., and Pindera, M.-J., "Local Field Effects in Titanium Matrix Composites Subjected to Fiber-Matrix Debonding," International Journal of Plasticity, Vol. 20, Nos. 8-9, 2004, pp. 1707-1737. doi:10.1016/j.ijplas.2003.11.019

[29] Ng, W. H., Salvi, A. G., and Waas, A. M., "Characterization of the In Situ Nonlinear Shear Response of Laminated Fiber-Reinforced Composites," Composites Science and Technology (in press).

A. Pelegri Associate Editor 